Meta

Journal des traducteurs

Translators' Journal

\title{
La terminologie de la cosmétologie
}

\section{Aline Francoeur, Elvira Martinez, Maryline Membré et Mamadou Samassa}

Volume 41, numéro 3, septembre 1996

URI : https://id.erudit.org/iderudit/002771ar

DOI : https://doi.org/10.7202/002771ar

Aller au sommaire du numéro

Éditeur(s)

Les Presses de l'Université de Montréal

ISSN

0026-0452 (imprimé)

1492-1421 (numérique)

Découvrir la revue

Citer cet article

Francoeur, A., Martinez, E., Membré, M. \& Samassa, M. (1996). La terminologie de la cosmétologie. Meta, 41(3), 419-434. https://doi.org/10.7202/002771ar

\section{Résumé de l'article}

Cet article présente les grandes étapes d'une recherche terminologique thématique effectuée par quatre étudiants en terminologie en vue de constituer un lexique bilingue dans le domaine de la cosmétologie. d'utilisation que vous pouvez consulter en ligne.

https://apropos.erudit.org/fr/usagers/politique-dutilisation/ 


\title{
LA TERMINOLOGIE DE LA COSMÉTOLOGIE
}

\author{
Aline Francoeur, Elvira Martinez, Marilyne Membré \\ ET MAMADOU SAMASSA
}

\begin{abstract}
Résumé
Cet article présente les grandes étapes d'une recherche terminologique thématique effectuée par quatre étudiants en terminologie en vue de constituer un lexique bilingue dans le domaine de la cosmétologie.
\end{abstract}

Abstract

Four graduate students present the main phases of a terminology research project which they conducted with a view to compiling a bilingual lexicon in the area of cosmetology.

La mode même et les pays règlent ce que l'on appelle beauté.

Pascal, Les passions de l'amour.

\section{INTRODUCTION}

C'est le propre de la nature humaine que de vouloir améliorer la beauté; l'être humain essaie, à l'aide de toutes sortes d'artifices, d'embellir ce que la nature lui offre. De nos jours comme vraisemblablement depuis toujours, les gens sont prêts à investir temps et argent pour paraître tout à leur avantage. Très vite, les entreprises ont compris ce besoin et ont répondu à la demande en présentant un choix extraordinaire de produits des plus divers.

Dans le cadre de notre étude terminologique, nous nous sommes intéressés à un secteur où la beauté occupe une grande place: la cosmétologie. Le développement rapide de ce secteur a déclenché la création d'une profusion de nouveaux produits cosmétiques dont les appellations et les qualités sont vantées par une importante diffusion publicitaire. Il faut notamment souligner l'importance de la publicité au point de vue linguistique : sa présence quotidienne et l'effet de répétition qu'elle crée souvent donnent vie aux mots et contribuent à les valoriser et à les faire accepter du public. Comme nous le verrons plus loin, cette forte présence de la publicité se traduit entre autres par un usage intensif d'adjectifs dans les dénominations des produits cosmétiques.

Après avoir procédé à une première exploration de la documentation existante, nous avons décidé de limiter notre recherche à un sous-domaine précis, la cosmétologie du visage, qui offre suffisamment matière à étude. Nous avons constitué notre dossier documentaire, pour ensuite procéder à son analyse en vue d'élaborer un lexique bilingue français-anglais. Nous avons cru bon d'inclure à ce lexique les collocations les plus fréquemment rencontrées lors du dépouillement terminologique.

Dans ce qui suit, nous résumons les grandes étapes de notre étude terminologique: la recherche documentaire, l'arbre de domaine, l'analyse des termes et des collocations retenus et la constitution de la fiche terminologique. À titre de résultat de notre étude, nous proposons un lexique bilingue français-anglais constitué de 279 termes et de 55 collocations (voir annexe 2). 


\section{LA RECHERCHE DOCUMENTAIRE}

Nous avons tout d'abord dressé un inventaire des publications dans le domaine de la cosmétologie et nous nous sommes procuré des catalogues de produits cosmétiques auprès de diverses maisons fabricantes de produits. Cela nous a permis d'élaborer une liste de documents composée d'ouvrages spécialisés, de manuels d'enseignement, de lexiques, de dictionnaires, de catalogues et de revues. Devant cette masse de documentation et surtout en raison du caractère disparate de celle-ci, nous avons procédé à certains regroupements, de façon à obtenir le classement suivant :

1) Documentation primaire: ouvrages de spécialistes, manuels d'enseignement et revues spécialisées.

2) Documentation secondaire : dictionnaires et encyclopédies, lexiques et glossaires.

3) Documentation publicitaire: catalogues de produits et publicités relevées dans diverses revues.

Nous avons ensuite procédé à la sélection des ouvrages qui nous semblaient les plus pertinents en fonction des critères suivants établis au préalable:

1) La date de l'ouvrage: nous avons retenu les publications les plus récentes car la cosmétologie est un domaine en constante évolution.

2) La qualité de la publication: nous avons sélectionné les ouvrages qui font preuve de rigueur dans la démarche méthodologique, dans le choix des termes de base, dans la précision des définitions et des contextes définitoires. En ce qui concerne les catalogues et les revues, nous avons privilégié les maisons de renom en partant du principe que, compte tenu des moyens dont elles disposent, elles peuvent atteindre un vaste public.

3) Les références bibliographiques: leur présence montre que le travail a fait l'objet d'une recherche soignée, ce qui donne un caractère fiable à l'ouvrage.

\section{L'ARBRE DE DOMAINE}

Notre étude de la cosmétologie nous a permis d'établir l'arbre de domaine suivant:

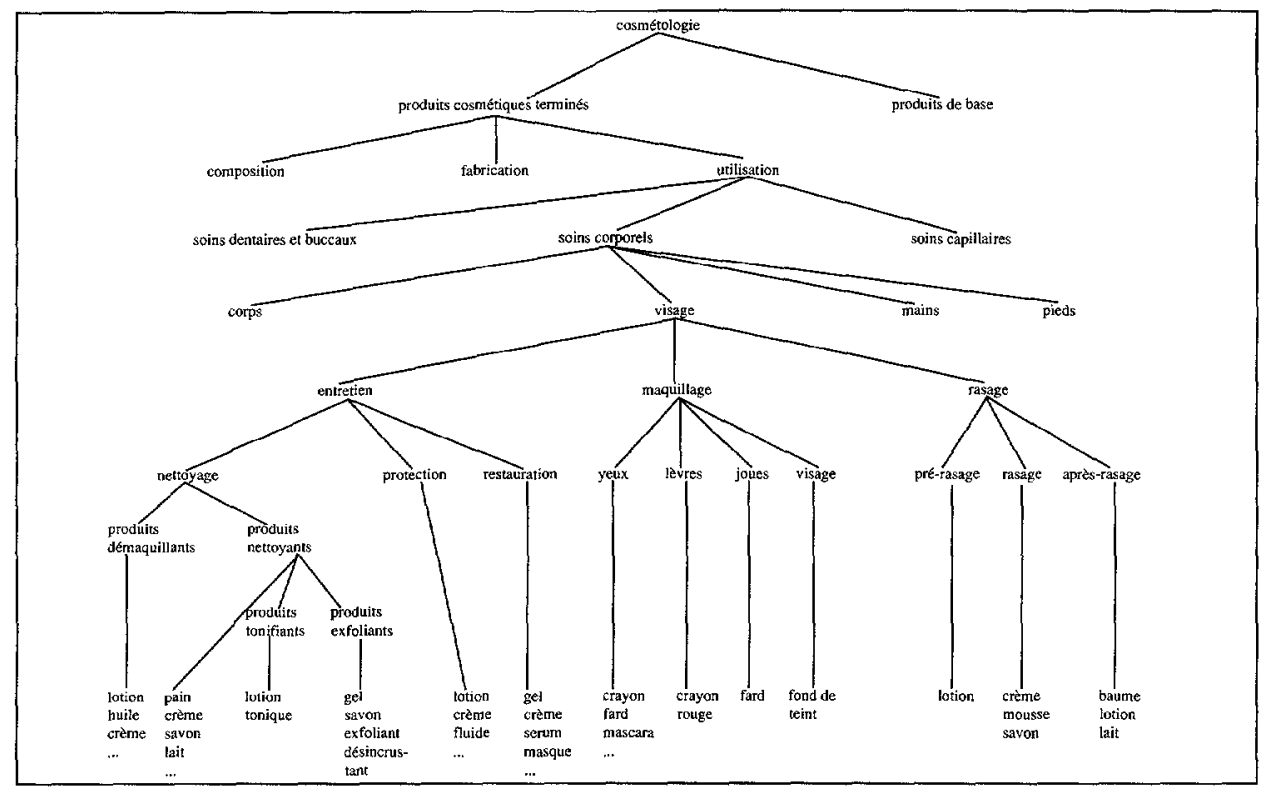


Les premières subdivisions de l'arbre sont inspirées de la définition de la cosmétologie relevée dans le Grand dictionnaire encyclopédique Larousse (1983: 2662-2663) : «Étude de tout ce qui se rapporte aussi bien aux produits cosmétiques terminés, à leur activité et à leur mode d'emploi qu'aux produits de base servant à leur préparation».

Nous voulions d'abord proposer deux grands types d'utilisation, soit l'hygiène corporelle d'une part et l'esthétique d'autre part. Cependant, la notion d'hygiène étant relative et variant selon les cultures, nous avons préféré abandonner cette division pour nous baser sur les trois types d'utilisation des produits mentionnés dans la plupart des définitions de la notion de produit cosmétique ${ }^{1}$ : les soins dentaires et buccaux, les soins corporels, et les soins capillaires.

Après avoir décidé de concentrer notre recherche sur les produits de soins corporels, nous avons constaté que ceux-ci appartiennent en fait à quatre catégories : ceux destinés aux soins du corps en général, aux soins du visage, au traitement des mains et au traitement des pieds.

Comme nous l'avons mentionné plus haut, nous nous sommes limité à l'étude des produits cosmétiques appartenant au sous-domaine des produits pour le visage, qui ont été divisés en trois catégories, selon leurs différentes utilisations: ENTRETIEN, MAQUILLAGE, RASAGE. Le nettoyage, la protection et la restauration constituent les trois étapes de l'ENTRETIEN de la peau. Sous nettoyage, nous avons classé les produits nettoyants, tels que définis par Hernandez (1992: 292) : «Préparations cosmétiques destinées à éliminer les impuretés poussiéreuses, les cellules mortes, la sueur, le sébum et éventuellement les traces de maquillages». Puis les produits démaquillants, qui possèdent aussi la propriété d'éliminer les impuretés et les sécrétions de la peau, mais qui, en plus, ont pour fonction spécifique d'enlever le maquillage. Nous avons par ailleurs divisé les produits nettoyants en deux types: les produits tonifiants, c'est-à-dire ceux qui parachève le nettoyage en éliminant les dernières traces de saleté ou de maquillage, et les produits exfoliants (ou gommants), qui visent à débarrasser la peau des cellules mortes afin de lui redonner un aspect propre.

La deuxième étape de l'ENTRETIEN consiste à protéger la peau contre la déshydratation, c'est-à-dire à empêcher l'évaporation de l'eau que la peau contient déjà, d'où la division protection dans l'arbre. Finalement, la restauration (ou restructuration) de la peau, qui vise à stimuler le renouvellement cellulaire et à restaurer l'élasticité et la tonicité de la peau, ce qui permet notamment de prévenir l'apparition de rides et de ridules, constitue la dernière étape de l'ENTRETIEN,

Les produits de MAQULLAGE ont été classés en fonction des parties du visage auxquelles ils sont principalement destinés : les yeux, les lèvres et les joues. Quant aux produits de RASAGE, ils ont été ordonnés selon les différentes étapes de l'opération de rasage elle-même : le pré-rasage, le rasage et l'après-rasage.

Par ailleurs, nous avons dressé une liste des produits cosmétiques de base (crème, lait, gel, etc.) qui, selon les éléments qui les composent, possèdent des fonctions et vertus différentes. Pour faciliter la compréhension et l'établissement des liens, nous proposons à l'annexe 1 les définitions ${ }^{2}$ de ces termes de base.

\section{ANALYSE DES TERMES}

L'analyse des 750 unités terminologiques relevées lors de la première étape du dépouillement nous a permis de constater que 20 termes servent de base pour la formation des termes complexes propres à notre domaine d'étude: baume, crayon, crème, émulsion, eye-liner, fard, fluide, fond de teint, gel, huile, lait, lotion, mascara, masque facial, mousse, pain, poudre, rouge à lèvres, savon, sérum. Les termes complexes 
correspondent aux matrices terminogéniques habituelles, la plus productive étant sans contredit $\mathbf{N}+\mathbf{A d j}$. Les adjectifs qui entrent en jeu dans cette matrice sont en nombre restreint et peuvent être classés en trois grandes catégories sémantiques : ceux qui réfèrent à l'action du produit (astringent, adoucissant, clarifiant, hydratant, démaquillant, désincrustant, exfoliant, nourrissant, protecteur, régénérateur, équilibrant, tonifiant, raffermissant, stimulant, tonique); ceux qui décrivent une caractéristique du produit (crémeux, teinté, gras, doux, moussant, coloré, alcoolisé) et ceux qui désignent la partie du corps sur laquelle le produit sera appliqué (facial, labial). Le tableau ci-dessous illustre la première catégorie sémantique d'adjectifs et montre que chacun des adjectifs peut être adjoint à une base différente pour former un nouveau terme.

\begin{tabular}{|c|c|c|c|c|}
\hline $\begin{array}{l}\text { TERME DE } \\
\text { BASE } \\
\end{array}$ & crème & lait & lotion & masque \\
\hline ADJECTIF & $\begin{array}{l}\text { hydratante } \\
\text { démaquillante } \\
\text { nettoyante } \\
\text { exfoliante } \\
\text { adoucissante } \\
\text { nourrissante } \\
\text { protectrice } \\
\text { désincrustante } \\
\text { régénératrice } \\
\text { équilibrante } \\
\text { raffermissante }\end{array}$ & $\begin{array}{l}\text { hydratant } \\
\text { démaquillant } \\
\text { nettoyant }\end{array}$ & $\begin{array}{l}\text { hydratante } \\
\text { démaquillante } \\
\text { nettoyante } \\
\text { exfoliante } \\
\text { adoucissante } \\
\text { astringente } \\
\text { clarifiante } \\
\text { purifiante } \\
\text { régénératrice } \\
\text { équilibrante } \\
\text { tonifiante } \\
\text { stimulante } \\
\text { tonique }\end{array}$ & $\begin{array}{l}\text { hydratant } \\
\text { nettoyant } \\
\text { exfoliant } \\
\text { adoucissant } \\
\text { nourrissant } \\
\text { astringent } \\
\text { purifiant } \\
\text { désincrustant }\end{array}$ \\
\hline
\end{tabular}

La matrice $\mathbf{N}+$ joncteur prépositionnel $+\mathbf{N}$ produit des termes dont le deuxième élément nominal réfère à une partie du visage, par exemple dans les termes ombre à paupières et fard à joues, à un moment d'utilisation (hydratant de jour, crème de nuit), à un élément constituant du produit (eau de lavande, lait de rose) ou encore à la consistance du produit (masque en crème, démaquillant en gel).

Les matrices de base admettent des expansions pour former des composés lourds du type masque rafraîchissant à peler aux fraises, lotion hydratante non parfumée pour peaux sensibles, crème à l'essence d'orchidée pour le dessous des yeux...

Par ailleurs, notre analyse fait ressortir l'influence publicitaire sur la création terminologique en cosmétologie. Nous avons notamment relevé des termes condensés, c'est-àdire formés par l'adjonction de deux éléments, unis ou non par un trait d'union (lotion tilleul, fard poudre, fard-crème, contour-yeux). Nous constatons également que de nombreux termes comportent un élément de dénomination particulier à une maison fabricante de produits. Par exemple, les termes mascara Opulencils et rouge à lèvres Coloriche sont propres à la maison Avon, les termes tonique floral Frâtcheur douce et fond de teint Douceur Naturelle, à la maison Yves Rocher, etc. Cette pratique semble avoir pour objectif de particulariser le produit en question par rapport au générique. 
Comme nous l'avons déjà mentionné, les unités terminologiques relevées proviennent principalement de documents de nature publicitaire. Chaque maison cherchant à distinguer ses propres marques, nous avons relevé un certain nombre de termes synonymes qu'il n'est pas toujours facile de différencier. Par exemple, les termes complexe, soin et traitement réfèrent à des produits qui sont en fait des crèmes, des laits ou des sérums. Ainsi, le soin du soir de la maison Yves Rocher est une crème de nuit, le complexe soin des yeux offert chez Avon est en fait une crème anti-rides. De même, le bâton cosmétique de la maison Avon, le bâton anti-cernes de Clinique et le dissimulateur de Cover Girl sont utilisés dans un même but, celui de camoufler les cernes et les imperfections cutanées. Les synonymes relevés correspondent généralement aux matrices suivantes :

N1 en N2 (démaquillant en gel) vs N1 + N2 (démaquillant gel). Les termes formés selon la matrice $\mathbf{N} 1$ en $\mathbf{N} 2$ sont plus répandus.

$\mathbf{N} 1$ à $\mathbf{N} 2$ (baume à lèvres) vs $\mathbf{N} 1$ pour $\mathbf{N} 2$ (baume pour les lèvres), où la structure N1 à $\mathbf{N} 2$ est la plus courante.

$\mathbf{N} 1$ pour $\mathbf{N} 2$ (exfoliant pour le visage) vs $\mathbf{N}+\mathbf{A d j}$ (exfoliant facial), où la première structure est la plus fréquente.

$\mathbf{N} 1$ de $\mathbf{N} 2$ (crème de nettoyage) vs $\mathbf{N}+$ Adj (crème nettoyante), la structure $\mathbf{N}+$ Adj étant sans contredit la plus répandue.

$\mathrm{N} 1$ anti $\mathbf{N} 2$ (crème anti-rides) vs $\mathbf{N 1}$ contre $\mathbf{N} 2$ (crème contre les rides), la tendance privilégiant la première construction, soit celle avec anti.

Par ailleurs, en vue de l'élaboration de notre lexique, nous avons dû procéder à l'élimination systématique de certaines catégories de termes. Dans cet esprit, nous avons écarté tous les termes purement publicitaires ainsi que ceux qui ne sont en fait qu'une énumération des éléments constituants du produit, du type crème gelée royale, gel nettoyant à base de boue. Nous avons également convenu de ne pas retenir les termes relatifs aux produits de bronzage, aux contenants des produits ou aux accessoires de maquillage pour nous concentrer uniquement sur les dénominations des produits pour le visage.

\section{LES COLLOCATIONS}

Dans le cadre de notre travail, nous considérons comme collocation un ensemble binaire de lexèmes ${ }^{3}$ partageant une affinité de sens, sans que leur combinatoire ne modifie leurs caractères sémantiques essentiels. Le sens global de la collocation est transparent (Clas 1994) ${ }^{4}$. La collocation se distingue de la combinaison libre par le fait que ses possibilités combinatoires sont restreintes.

La notion de collocation définie, nous avons procédé au dépouillement des sources afin de relever les collocations propres au domaine étudié. Le lexique en annexe comporte 58 collocations verbales, principalement du type verbe + complément d'objet direct. On constate que ces collocations décrivent généralement des propriétés d'action du produit: estomper les rides, resserrer les pores, dissimuler les imperfections. Le nom du produit en question joue le rôle de sujet du verbe de la collocation. Nous avons également relevé quelques collocations verbales dont la base est un verbe et le collocateur, le nom d'un produit, par exemple les collocations appliquer le fond de teint, estomper la poudre, qui réfèrent au mode d'application du produit.

\section{LA FICHE TERMINOLOGIQUE}

Nous avons étudié divers modèles de fiches et, comme il sied, nous avons conçu la nôtre en fonction de nos besoins. Compte tenu de la nature de notre travail et des résultats 
auxquels nous voulions parvenir, nous avons choisi un modèle de fiche à trois niveaux (information en français, en anglais, et d'ordre général), comportant des rubriques obligatoires et d'autres facultatives. Comme spécificité, nous avons introduit une rubrique collocation(s) en français et en anglais. Cette rubrique montre les collocateurs susceptibles d'être utilisés avec le terme qui fait l'objet de la fiche..

\begin{tabular}{|c|c|}
\hline Ent. française : & rouge à lèvres [a] \\
\hline Cat. gramm.: & $\mathrm{nm}$ \\
\hline $\begin{array}{l}\text { Variante(s): } \\
\text { Synonyme(s): }\end{array}$ & rouge $[b]$ \\
\hline Définition : & $\begin{array}{l}\text { Ils [les rouges à lèvres] sont constitués de corps gras, de cires, de } \\
\text { colorants et parfois de parfums. De consistance souple, ils ne doivent } \\
\text { pas se déformer par temps chaud. La teinte du rouge à lèvres doit se } \\
\text { transmettre à la lèvre et ne doit pas se modifier à la longue. [a] }\end{array}$ \\
\hline Contexte: & $\begin{array}{l}\text { Appliquer le rouge à lèvres avec un pinceau. Pour avoir la main plus } \\
\text { sûre, appuyer l'auriculaire replié sur le menton. [b] }\end{array}$ \\
\hline $\begin{array}{l}\text { Collocation(s): } \\
\text { Note(s) }\end{array}$ & appliquer le rouge à lèvres. \\
\hline $\begin{array}{l}\text { Ent. anglaise: } \\
\text { Variante(s): } \\
\text { Synonyme(s): }\end{array}$ & lipstick [c] \\
\hline Définition: & $\begin{array}{l}\text { Lipstick is a mixture of waxes, oils, and other emollients plus colors, } \\
\text { flavors, perfumes, and preservatives. Optional ingredients include } \\
\text { sunscreens, anti-irritants and antiseptics. [d] }\end{array}$ \\
\hline Contexte: & $\begin{array}{l}\text { When it comes to makeup, lipstick is on the most-wanted list. If } \\
\text { you're like the majority of women, it's the most frequently used item } \\
\text { in your bag of beauty tricks. [e] }\end{array}$ \\
\hline $\begin{array}{l}\text { Collocation(s): } \\
\text { Note(s): }\end{array}$ & to apply lipstick \\
\hline Voir aussi : & \\
\hline Sources: & $\begin{array}{l}\mathrm{a}=\text { Faster et Develle }(1984: 256) ; \mathrm{b}=\text { The Mary Kay Guide to Beauty } \\
(1983: 83) ; c=\text { Winter }(1974: 136) ; \mathrm{d}=\text { Arpel }(1986: 120) ; \mathrm{e}=\text { Revlon } \\
\text { Art of Beauty }(1982: 48) .\end{array}$ \\
\hline Domaine : & cosmétologie \\
\hline Rédacteur: & $\mathrm{AF}$ \\
\hline Date: & 1995.04 .14 \\
\hline
\end{tabular}




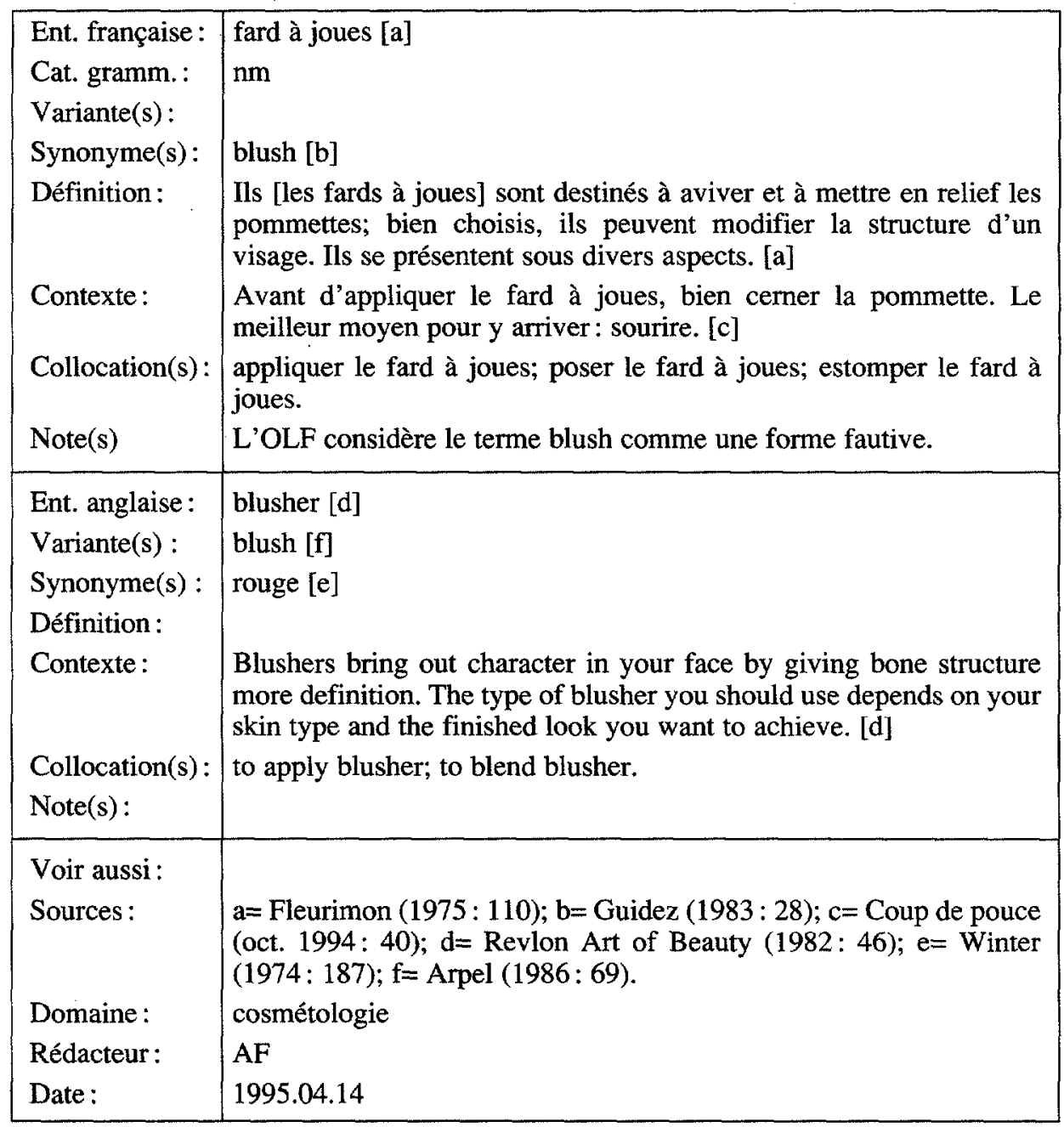

\section{CONCLUSION}

Notre recherche nous a permis de constater que la cosmétologie constitue un domaine très riche du point de vue terminologique. Nous avons notamment remarqué que la terminologie du domaine n'évolue pas seulement en fonction des progrès scientifiques et techniques, mais plus souvent en fonction d'opérations de marketing menées par les fabricants de produits cosmétiques. De plus, malgré l'évolution constante du domaine et l'apparition continuelle de nouveaux produits sur le marché, nous constatons que les constituants de base des produits restent les mêmes: il s'agit toujours de crèmes, de lotions, de laits, de poudres, etc. Du point de vue terminologique, il en découle que les unités complexes sont généralement formées d'un terme de base auquel on ajoute des qualificatifs qui désignent souvent les propriétés spécifiques du produit. On cherche ainsi à le différencier des produits concurrents et à attirer l'attention du public. De ce fait, on peut dire que la cosmétologie constitue une langue de spécialité bien structurée dont la 
principale particularité est de posséder un système de création néologique quasi inépuisable.

Notes

1. Hernandez (1992) propose notamment la définition suivante: «Sont compris comme produits cosmétiques ou produits d'hygiène corporelle, toutes les substances ou préparations autres que les médicaments destinées à être mises en contact avec les diverses parties superficielles du corps humain ou avec les dents et les muqueuses, en vue de les nettoyer, de les protéger, de les maintenir en bon état, d'en modifier l'aspect, de le parfumer ou d'en corriger l'odeur».

2. Les définitions que nous proposons sont inspirées de définitions ou de contextes définitoires relevés dans les ouvrages spécialisés consultés.

3. L'appellation des éléments de la collocation varie en fonction des auteurs. Par exemple, Hausmann parle de base et de collocateur, Mel'čuk de mot clé et de valeur de fonction lexicale. Pour les besoins du présent article, nous adoptons la typologie d'Hausmann.

4. Clas, A. (1994) : «Collocations et langues de spécialité, Meta, 39 (4), pp. 576-580.

\section{RÉFÉRENCES}

Documentation primaire

FLEURIMON, Jean-Pierre (1975) : Produits de beauté sans mystère: introduction à la cosmétologïe, Paris, Maloine, $215 \mathrm{p}$.

GODEAU, M.-J. et R. VILAIN (1984) : Les soucis esthétiques de la peau du visage, Paris, Masson, $190 \mathrm{p}$

HARRY, Ralph G. (1973) : Harry's Cosmetology, London, Leonard Hill Books, 824 p.

HERNANDEZ, Micheline et Marie-Madeleine MERCIER-FRESNEL (1988) : Dossier de l'esthétique cosmétique. Préparation aux examens d'État, Paris, Maloine.

POELMAN, M.C. (1987) : Initiation à la cosmétologie pratique, Paris, Technique et Documentation Lavoisier, $141 \mathrm{p}$.

RINZLER, Carol Ann (1977) : Cosmetics: What the Ads Don't Tell You, New York, Thomas Y. Crowell Company, 212 p.

THIERS, Henri (1962) : Les cosmétiques. Pharmacologie et biologie, Paris, Masson et Cie Editeurs, 300 p.

WINTER, Ruth (1974) : A Consumer's Dictionary of Cosmetic Ingredients, New York, Crown Publishers Inc., $236 \mathrm{p}$.

Documentation secondaire

ARPEL, Adrien (1986) : Adrien Arpel's 851 Fast Beauty Fixes and Facts, New York, Dell Publishing Co., $240 \mathrm{p}$.

Être au naturel présent : maquillage, Paris, Compagnie Internationale du Livre, 1986, 48 p.

FASTER, S. et D. DEVELLE (1984) : 500 conseils pour votre beauté, Paris, Larousse, $327 \mathrm{p}$.

GHEDIN, Josette (1976): Peau nue, moderne et vraie. Dernières techniques dasn le soin de la peau, Montréal, Alain Stanké, $192 \mathrm{p}$.

Grand dictionnaire encyclopédique Larousse (1983), Paris, Librairie Larousse.

GUIDEZ, Guylaine (1983) : Beauté et santé de votre peau. Les soins du visage, des cheveux et du corps, Paris, Solar.

HUTTON, Deborah (1984) : Vogue Complete Beauty, London, Octopus Books Limited, 224 p.

Revlon Art of Beauty, New York, Dolphin Books, 1982, 135 p.

The Mary Kay Guide to Beauty. Discovering your Special Look, California, Addison-Wesley, $1983,240 \mathrm{p}$.

The New Encyclopedia Britannica, Macropedia, Knowledge in Depth, Chicago, Encyclopedia Britannica, 1991.

VIDART, Rosine (1982) : Guide Rosine Vidart des produits de beauté, Paris, Olivier Orban, $381 \mathrm{p}$.

Documentation publicitaire

Catalogues de produits

Catalogue des produits Aloette, 1993.

Catalogue des parfumeries Dans un jardin.

Catalogue Avon.

Catalogue des produits Évolution et Odélys de Guerlain.

Catalogue Le Body Shop des produits pour la peau et les cheveux.

Catalogue des produits de beauté Nectar de La Baie.

Catalogue des produits Clinique.

Catalogue des produits Neostrata.

Catalogue des produits Nuskin.

Catalogue des produits Estée Lauder.

Guide des produits Orlane, Paris.

Guide Clarins des produits de beauté. 
Guide des produits L'Oréal Plénitude.

Guide des produits Vichy.

Le livre vert de la beauté 1995, Catalogue des produits Yves Rocher, France.

Manuel technique, Christian Dior, Paris.

Revues

Châtelaine

Coup de pouce

Elle

Flare

Santé

\section{ANNEXE 1 : DÉFINITIONS DES TERMES DE BASE EN COSMÉTOLOGIE}

BAUME : Produit de maquillage, souvent transparent, qui donne un léger brillant aux lèvres. Il peut se porter seul ou sous un rouge à lèvres.

CRAYON : $\grave{A}$ base de cires, de paraffine et de colorants, enrobé dans un étui la plupart du temps en bois. La mine est plus ou moins tendre.

ÉMULSION: Mélange d'eau et d'huile, système de deux phases liquides (huile et eau) dont l'une est finement dispersée dans l'autre.

EYE-LINER : Produit liquide ou compact destiné à souligner les paupières supérieure et inférieure de façon à intensifier le maquillage, à corriger ou à modifier la forme de l'œil.

FARD : Produit de maquillage destiné à masquer certaines irrégularités de la peau, à rehausser l'éclat du teint ou à modifier la couleur de la peau.

FLUIDE : Émulsion très liquide.

FOND DE TEINT : Émulsion colorée et grasse destinée à unifier le teint, à accentuer ou à estomper les reliefs du visage et à masquer les imperfections de la peau.

GEL : Produit cosmétique obtenu à partir de collö̈des, c'est-à-dire de substances épaisses émulsionnées dans l'eau et très concentrées, s'apparentant à la colle par leur structure physique.

HUILE: Substance formée principalement d'acides gras d'origine végétale, animale, minérale, ou synthétique et servant de base aux émulsions.

LAIT ET CRÈME : Émulsion huile dans eau ou eau dans huile; la différence entre lait et crème réside dans la fluidité du produit. Les laits et les crèmes peuvent être utilisés pour le nettoyage, l'embellissement ou la protection.

LOTION (ou TONIQUE) : Solution aqueuse, généralement composée d'huiles essentielles et d'eau distillée, souvent colorée. La lotion s'utilise généralement pour terminer l'étape du démaquillage.

MASCARA : Produit pour les cils qui se présente sous forme de crème ou de liquide. Il sert à épaissir, à allonger, à colorer, à nourrir et à protéger les cils.

MASQUE FACIAL: Préparation sous forme de crème, poudre, pâte ou gelée, destinée à lisser la peau du visage et à faire tenir le maquillage. Ses effets sont fonction des ingrédients actifs qu'il contient.

MOUSSE : Émulsion présentée en conditionnement pressurisé.

PAIN : Produit de nettoyage présenté en barre rectangulaire. Son pH est faible ou neutre, il ne mousse presque pas.

POUDRE: Substance solide, souvent parfumée, divisée en très petites particules et composée de talc, d'oxyde de zinc et d'amidon. Elles sont destinées à donner un aspect mat et velouté au visage.

ROUGE À LÈVRES : Produit composé d'un mélange de différents excipients destiné à donner aux lèvres une couleur attrayante. Il peut de plus permettre de modifier la forme ou le volume des lèvres.

SAVON : Fait d'acides gras, obtenus par saponification en faisant agir une base minérale, soude ou potasse sur un mélange d'esters d'acides gras d'origine animale ou végétale.

SÉRUM : Extrait de substances actives à l'état pur (isolees de tissus humains, animaux ou végétaux). Selon sa particularité, le sérum resserre la peau, la stimule, la rend plus lisse ou plus fraîche.

\section{ANNEXE 2: LEXIQUE BILINGUE FRANÇAIS-ANGLAIS}

Le lexique que nous proposons est composé de 279 termes et de 55 collocations. Dans le cas de synonymes ou de variantes orthographiques, seul le terme le plus fréquent a été traité. Les variantes orthographiques ou les synonymes moins fréquents renvoient au terme traité. Par exemple, masque facial renvoie à masque pour le visage, anticernes renvoie à anti-cernes, etc.

\section{Termes français}

anti-cerne (v. anti-cernes)

anti-cernes

anticernes ( $v$, anti-cernes)

après-rasage

astringent

\section{Termes anglais}

concealer

after shave

astringent 
astringent clarifiant

base hydratante

base protectrice pour les lèvres

base protectrice pour les paupières

bâton anti-cernes

bâton cosmétique (v. bâton anti-cernes)

baume

baume à lèvres

baume après-rasage

baume contour des yeux

baume hydratant pour les lèvres

baume pour les lèvres (v. baume à lèvres)

blush (v. fard)

blush gel

brillant à lèvres

cache-cernes ( $v$. anti-cernes)

cold-cream

cold-cream à rincer

crayon à lèvres

crayon à sourcils

crayon automatique pour les lèvres

crayon automatique pour les yeux

crayon contour des lèvres (v. crayon à lèvres)

crayon contour des yeux

crayon contour lèvres (v. crayon à lèvres)

crayon kajal

crayon khôl

crayon ligneur

crayon ombre-ligneur pour les yeux

(v. crayon ombreur-ligneur)

crayon ombreur-ligneur

crayon pour les lèvres (v. crayon à lèvres)

crayon pour les sourcils

crayon pour les yeux

crayon-traceur à lèvres (v. crayon à lèvres)

crème

crème à raser

crème adoucissante

crème antirides

crème contour des yeux

crème contre les rides (v. crème antirides) crème de base teintée

crème de démaquillage

(v. crème démaquillante)

crème de gommage (v. crème gommante)

crème de jour

crème de jour fluide

crème de jour hydratante

crème de jour rajeunissante

crème de jour teintée

crème de nettoyage (v. crème nettoyante)

crème de nuit clarifying astringent

moisture base

lip protecting base

eye protecting base

concealing stick

balm

lip balm

after shave balm

eye contour balm

moisturizing lip balm

gel blusher

gloss

lip gloss

cold cream

water rinsable cold cream

lip pencil

lip liner pencil

lip-liner pencil

eyebrow pencil

brow definer

automatic lip contour pencil

automatic eye contour pencil

eye-defining pencil

eye definer

kajal pencil

kohl pencil

eye-liner pen

eyeshadow lining pencil

eyebrow pencil

brow definer

eye pencil

cream

shaving cream

soothing cream

anti-wrinkle cream

wrinkle defense cream

eye care cream

eye beauty cream

tinted foundation cream

day cream

daytime moisture cream

daytime fluid moisturizer

moisturizing day cream

rejuvenating cream

tinted moisturizer

night cream 
crème de nuit régénératrice

crème de soin équilibrantere

crème de soins hydratante teintée

crème démaquillante

crème démaquillante hydratante

crème désincrustante (v. crème gommante)

crème éclaircissante

crème équilibrante

crème évanescente

crème exfoliante

crème gommante

crème hydratante

crème hydratante teintée

(v. crème de jour teintée)

crème nettoyante à rincer

crème nettoyante

crème nourrissante

crème nutritive

crème pour les rides

crème raffermissante

crème régénératrice

crème régénératrice pour les yeux crème rénovatrice (v. crème tégénératrice) crème teintée contre les imperfections démaquillant

démaquillant à rincer

démaquillant en gel

démaquillant fluide

démaquillant liquide

démaquillant mousse

démaquillant pour les yeux

démaquillant purifiant

démaquillant-gel (v. démaquillant en gel)

désincrustant facial

(v. désincrustant pour le visage)

désincrustant pour le visage

beau florale

émulsion

émulsion démaquillante

émulsion hydratante

exfoliant

exfoliant facial (v. exfoliant pour le visage)

exfoliant pour le visage

eye-liner

eye-liner en pain

eye-liner en poudre (v. eye-liner en pain)

eyeliner liquide

fard

fard à joues replenishing night cream

balancing moisture cream

revitalizing tinted moisturizer

cleansing cream

hydrating cleansing cream

banishing cream

equalizing moisture cream

vanishing cream

exfoliating cream

cleansing cream scrub

moisture cream

moisturizing cream

creamy wash-off cleanser

cleansing cream

nourishing cream

nutritive cream

anti-wrinkle cream

wrinkle defense cream

refirming cream

replenishing cream

regenerative cream

replenishing eye cream

tinted blemish cream

make-up remover

cleanser

cosmetic remover

cleansing wash

cleansing gel

cleansing liquid

liquid make-up remover

cleansing liquid

liquid make-up remover

foaming cleanser

eye make-up remover

eye makeup remover

oil removing cleanser

face scru

floral water

emulsion

cleansing emulsion

cleansing lotion

moisturizing emulsion

scrub

facial scrub

eyeliner

eye liner

cake eyeliner

liquid eyeliner

blush

blusher

rouge

blush 
fard à joues compact (v. fard à joues en poudre) fard à joues crème (v. fard à joues en crème) fard à joues en crème

cream rouge

fard à joues en gel

fard à joues en poudre

fard à joues en poudre compacte

(v. fard à joues en poudre)

fard à joues en poudre pressée

(v. fard à joues en poudre)

fard à joues fluide

fard à joues gras

fard à joues liquide

fard à paupière

fard à paupières compact

fard à paupières crème

fard à paupières crème poudre

fard à paupières en bâton

fard à paupières en crème

fard à paupières en poudre pressée

fard stick

fluide hydratant

fluide hydratant non gras

fond de teint

fond de teint compact

fond de teint crème (v. fond de teint en crème)

fond de teint en bâton

fond de teint en crème

fond de teint fluide

fond de teint fluide hydratant

fond de teint gel

fond de teint gras

fond de teint hydratant

fond de teint liquide

fond de teint liquide non gras

fond de teint mat

fond de teint matifiant

fond de teint poudre-crème

fond de teint raffermissant

fond de teint sec

liquid rouge

cream rouge

cream blusher

liquid rouge

seye shadow

eyelid shadow

eyeshadow

eye color

brush-on eyeshadow

cream eye shadow

cream eyeshadow

cream powder eyeshadow

eyeshadow stick

cream eye shadow

cream eyeshadow

brush-on eyeshadow

blush stick

moisture fluid

oil-free moisture lotion

foundation

face makeup

foundation makeup

make-up foundation

pancake makeup

cake foundation

cake make-up

stick make-up

stick foundation

cream foundation

fluid foundation

fluid moisturizing foundation

gel foundation

oil make up

oil foundation

hydrating foundation

moisturizing foundation

liquid foundation

liquid make-up

liquid makeup

oil-free liquid foundation

matte finish makeup

matte finish foundation

oil control foundation

cream-powder base

firming foundation

dry cake make-up

gel 
gel apaisant

gel contour des yeux

gel démaquillant

gel équilibrant

gel exfoliant

gel hydratant

gel normalisant

gel rafraîchissant pour les yeux

gel réparateur

glosses

huile

huile démaquillante

huile démaquillante pour les yeux

hydratant

hydratant de jour

hydratant non gras

hydratant teinté

lait

lait après-rasage

lait de beauté

lait de démaquillage

lait de toilette

lait démaquillant

lait hydratant

lait nettoyant

lotion

lotion à nettoyer (v, lotion nettoyante)

lotion adoucissantes

lotion adoucissante pour la peau

lotion après-rasage

lotion astringente

lotion avant-rasage

lotion clarifiante

lotion démaquillante

lotion démaquillante pour les yeux

lotion équilibrante

lotion exfoliante

lotion faciale

lotion florale

lotion hydratante

lotion nettoyante

lotion nettoyante astringente

lotion pour le visage

lotion pré-rasage

lotion régénératrice

lotion stimulante

lotion tonifiante (v. lotion tonique)

lotion tonique

lotion tonique régulatrice

lotion tonique sans alcool

mascara

mascara à l'épreuve de l'eau enhancing gel

eye contour gel

cleansing gel

balancing gel

foaming scrub

moisturizing gel

rebalancing gel

refreshing eye gel

repairing gel

gloss

lip gloss

oil

cleansing oil

eye cleansing oil

moisturizer

daytime moisturizer

oil-free moisturizer

tinted moisturizer

milk

after-shave milk

beauty make-up milk

beauty milk

cleansing milk

beauty lotion

cleansing milk

moisturizing lotion

cleansing milk

lotion

oothing lotion

skin soothing lotion

after-shave lotion

astringent lotion

pre-shaving lotion

pre-shave lotion

preshave lotion

clarifying lotion

make-up remover lotion

eye make-up remover lotion

skin conditioner

exfoliating lotion

skin clarifier

facial lotion

floral lotion

moisturizing toner

moisture lotion

cleansing lotion

astringent cleansing lotion

facial lotion

pre-shaving lotion

pre-shave lotion

preshave lotion

replenishing lotion

stimulating lotion

toning lotion

toner

oil-control toner

alcohol-free toner

mascara

waterproof mascara 
mascara crème (v. mascara en crème)

mascara en cake

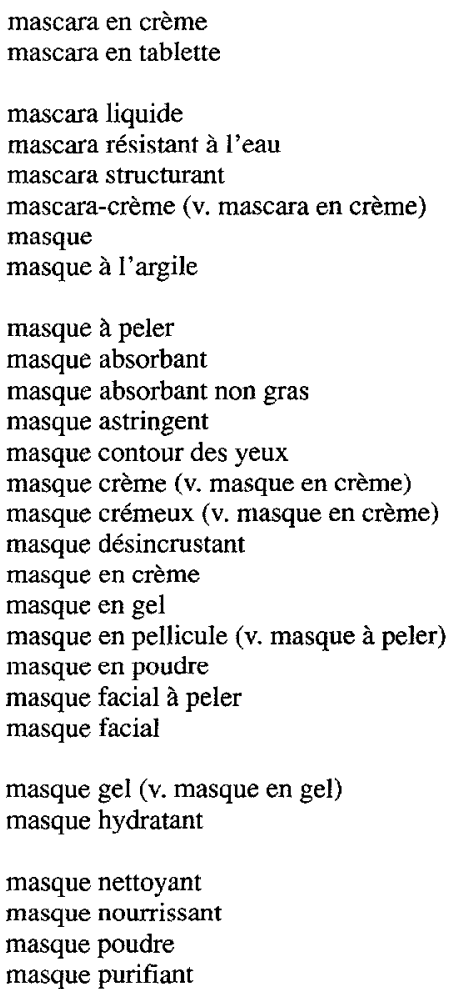

block mascara
cake mascara

cream mascara

block mascara

cake mascara

liquid mascara

waterproof mascara

conditioning mascara

mask

argillaceous mask

clay mask

peel-off mask

absorbant mask

oil-free refining mask

astringent mask

eye contour mask

exfoliating mask

cream mask

gel mask

powder mask

peel-off facial mask

facial mask

face mask

moisturizing mask

moisture mask

cleansing mask

nourishing mask

powder mask

cleansing mask

purifying mask

conditioning mask

treatment mask

shaving foam

cleansing foam

fresh foaming cleanser

active cleansing foam

cleanser

foaming cleanser

foaming facial cleanser

eye shadow

eyelid shadow

eyeshadow

eye color

cream eye shadow

cream eyeshadow

brush-on eyeshadow

syndet

cleansing cake

cleansing bar

hydrating cleansing bar

ointment

powder 
poudre compacte

poudre compacte non grasse

poudre compacte pour le visage

poudre libre

poudre libre pour le visage

poudre libre translucide

poudre pressée

poudre translucide

rimmel

rouge (v. rouge à lèvres)

rouge à lèvres

rouge à lèvres indélébile

(v. rouge à lèvres longue durée)

rouge à lèvres longue durée

rouge à lèvres modifiant

savon à barbe (v. savon à raser)

savon à barbe en poudre

savon à barbe liquide

savon à raser

savon exfoliant

savon facial

sérum

sérum multi-tenseur raffermissant

sérum raffermissant

sérum remodelant

stimulant cellulaire

stylo mousse anti-cernes

syndet

tonifiant (v. tonique)

tonifiant clarifiant

tonifiant régénérateur

tonique

tonique adoucissant (v. lotion adoucissante)

tonique astringent

tonique doux

tonique floral

tonique purifiant

\section{Collocations françaises}

adoucir la peau rasée de près

adoucir la surface de la peau

adoucir les lèvres

allonger les cils

apaiser la peau

appliquer l'anti-cernes

appliquer l'ombre à paupières

appliquer la poudre

appliquer le fard à joues

appliquer le fond de teint

appliquer le mascara

appliquer le masque facial

appliquer le rouge à lèvres

calmer l'épiderme

camoufler les cernes

camoufler les décolorations de la peau face powder

compact powder

oil free compact powder

pressed face powder

loose powder

loose face powder

translucent loose powder

pressed powder

translucent face powder

translucent powder

block mascara

cake mascara

lipstick

lip colour

long-lasting lipstick

lip colour modifier

powdered shaving soap

liquid shaving soap

shaving soap

cleansing bar

facial soap

serum

skin firming concentrate

firming serum

retexturing serum

cell energizing formula

foam tipped concealer pen

syndet

skin clarifier

replenishing toner

toner

skin toner

astringent lotion

mild toner

mild skin toner

floral toner

purifying toner

Collocations anglaises

to soothe just-shaved skin

to refine skin surface

to soften lips

to lengthen lashes

to relieve the skin

to apply concealer

to apply eye shadow

to apply face powder

to apply blusher

to apply foundation

to apply mascara

to apply the facial mask

to apply lipstick.

to apply lip colour

to soothe the epidermis

to conceal dark circles

to conceal discolourations 
camoufler les imperfections

colorer les cils

courber les cils

définir les sourcils

dégorger les pores

dessiner les lèvres

dissimuler les cernes

dissimuler les décolorations de la peau

dissimuler les imperfections

éliminer l'excès de sébum

éliminer les cellules mortes

enlever l'excès d'huile

enlever l'excès de sébum

enlever la saleté incrustée

enlever les cellules mortes

enlever les peaux mortes

(v. enlever les cellules mortes)

épaissir les cils

estomper le fard à joues

estomper le fond de teint

fixer le maquillage

hydrater les lèvres

nettoyer en profondeur

poser l'ombre à paupières

protéger l'épiderme

protéger les lèvres

purifier la peau

rafraîchir l'épiderme

rafraîchir la peau

rafraîchir la surface de la peau

réduire le brillant

réduire les bouffissures

réduire les cernes

renforcer les cils

resserrer les pores (de la peau)

revitaliser les cils

séparer les cils

souligner les yeux

stimuler la circulation

tracer les yeux to conceal blemishes

to colour lashes

to curl eyelashes

to define eyebrow

to unclog pores

to define lips

to hide dark circles

to hide skin discolourations

to hide blemishes

to remove excess oil

to remove dead cells

to remove excess oil

to remove excess sebum

to remove embedded dirt

to remove dead skin cells

to thicken lashes

to blend blusher

to blend foundation

to set makeup

to moisturize lips

to deep clean pores

to apply eye shadow

to protect the epidermis

to protect lips

to refine the skin

to refresh skin

to freshen the skin

to refresh skin

to freshen skin surface

to reduce shine

to reduce puffiness

to reduce dark circles

to strengthen lashes

to tighten pores

to condition lashes

to separate lashes

to define the eyes

to stimulate circulation

to shape the eyes 